\title{
SEMIGROUPS SATISFYING A STRONG FØLNER CONDITION
}

\author{
L. N. ARGABRIGHT AND C. O. WILDE ${ }^{1}$
}

1. Introduction. Let $S$ be a discrete semigroup and let $m(S)$ denote the Banach space (under the supremum norm) of all bounded realvalued functions on $S$. If $f \in m(S)$ and $s \in S$ then $l_{s} f$ and $r_{s} f$ are the functions in $m(S)$ defined by $l_{s} f(t)=f(s t)$ and $r_{s} f(t)=f(t s)$. Any element $\mu \in m(S)^{*}$ which is positive and has norm 1 will be called a mean on $m(S)$. A mean $\mu$ is left [right] invariant if $\mu\left(l_{s} f\right)=\mu(f)\left[\mu\left(r_{f} f\right)\right.$ $=\mu(f)]$ for all $f \in m(S)$ and all $s \in S$. A semigroup $S$ may or may not admit a left [right] invariant mean; those which do are called left [right] amenable.

Another Banach space associated with $S$ is the space $l_{1}(S)$ of all real-valued functions $g$ on $S$ such that $\sum\{|g(s)|: s \in S\}<\infty$, the latter quantity being defined to be $\|g\|$. Convolution is defined in $l_{1}(S)$ by the formula

$$
g_{1} \cdot g_{2}(t)=\sum\left\{g_{1}\left(s_{1}\right) g_{2}\left(s_{2}\right): s_{1} s_{2}=t\right\}
$$

and, under this multiplication, $l_{1}(S)$ becomes a Banach algebra. There is a natural linear isometry $\tau: l_{1}(S) \rightarrow m(S)^{*}$ defined by $g \rightarrow \tau_{g}$ where $\tau_{0}(f)=\sum\{f(s) g(s): s \in S\} \quad\left(f \in m(S), g \in l_{1}(S)\right)$. We can (and shall) regard $l_{1}(S)$ as being embedded in $m(S)^{*}$ via this mapping. Note that an element $g$ of $l_{1}(S)$ induces a mean on $m(S)$ if and only if $g(t) \geqq 0$ for all $t$ and $\sum\{g(t): t \in S\}=1$. Such an element of $l_{1}(S)$ is called a finite mean if $\{t: g(t)>0\}$ is finite. If $A$ is a nonvoid finite subset of $S$ then the function

$$
\begin{aligned}
\mu_{A}(t) & =1 /|A| & & t \in A, \\
& =0 & & t \in A,
\end{aligned}
$$

will be called an arithmetic mean on $S .{ }^{2}$

We shall also want to regard $S$ as being embedded in $l_{1}(S)$ via the mapping $s \rightarrow I(s)$ where

Received by the editors November 1, 1966 and, in revised form, November 21, 1966. tion.

1 The authors gratefully acknowledge the support of the National Science Founda-

${ }^{2}$ In general, $|M|$ will denote the cardinality of a (finite) set $M$. 


$$
\begin{array}{rlrl}
I(s)(t)=1 & & \text { if } s=t, \\
& =0 & & \text { if } s \neq t .
\end{array}
$$

We shall not distinguish between $s$ and $I(s)$ and hence write $s \cdot g$ in place of $I(s) \cdot g\left(g \in l_{1}(S)\right)$.

Theorem (M. M. Day [4]). A semigroup $S$ is left amenable if and only if there is a net $\left\{g_{\gamma}\right\}$ of finite means on $S$ which converge in norm to left invariance: $\lim \left\|s \cdot g_{\gamma}-g_{\gamma}\right\|=0$ for all $s \in S$.

See [7], Theorem 2.2, for an elegant proof of Day's theorem.

2. The strong F $\varnothing l$ lner condition. According to a theorem of F $\varnothing$ lnerFrey (see [7]) a left amenable semigroup must satisfy the following condition:

(FC) Given a finite set $F \subset S$ and $\epsilon>0$, there exists a finite set $A \subset S$ such that $|s A \sim A|<\epsilon|A|$ for all $s \in F$.

This condition is, however, not sufficient for a semigroup to be left amenable. In fact, any finite semigroup $S$ satisfies (FC) (take $A=S$ ) and there are finite semigroups which are not left amenable.

If $S$ satisfies the left cancellation law then clearly $|s A \sim A|$ $=|A \sim s A|$ for any finite set $A \subset S$ and $s \in S$. Thus, in this case, the condition $(\mathrm{FC})$ is equivalent to

(SFC) Given a finite set $F \subset S$ and $\epsilon>0$, there exists a finite set $A \subset S$ such that $|A \sim s A|<\epsilon|A|$ for all $s \in F$.

The condition (SFC) is, in any case, a sufficient condition for a semigroup to be left amenable. In fact, (SFC) implies an even stronger conclusion: There exists a net of arithmetic means converging in norm to left invariance.

Lemma 1. If $A$ is a finite subset of $S$ and $s \in S$, then $\left\|s \cdot \mu_{A}-\mu_{A}\right\|$ $=2|A \sim s A| /|A|$ for all $s \in S$.

Proof. If $B \subset S$ and $s \in S$, let $s^{-1} B=\{x \in S \mid s x \in B\}$. We will write $s^{-1} t$ in place of $s^{-1}\{t\}$. Then

$$
\begin{aligned}
\left(s \cdot \mu_{A}-\mu_{A}\right)(t) & =\left|A \cap s^{-1} t\right| /|A| & & \text { if } t \in s A \sim A, \\
& =-1 /|A| & & \text { if } t \in A \sim s A, \\
& =\left(\left|A \cap s^{-1} t\right|-1\right) /|A| & & \text { if } t \in A \cap s A, \\
& =0 & & \text { otherwise, }
\end{aligned}
$$

and hence 


$$
\begin{aligned}
\left\|s \cdot \mu_{A}-\mu_{A}\right\|= & \sum_{t \in s A \sim A} \frac{\left|A \cap s^{-1} t\right|}{|A|}+\frac{|A \sim s A|}{|A|} \\
& +\sum_{t \in A \cap s A} \frac{\left|A \cap s^{-1} t\right|-1}{|A|} \\
= & \sum_{t \in s A} \frac{\left|A \cap s^{-1} t\right|}{|A|}+\frac{|A \sim s A|}{|A|}-\frac{|A \cap s A|}{|A|} \\
= & \frac{1}{|A|}\{|A|+|A \sim s A|-|A \cap s A|\} \\
= & \frac{2|A \sim s A|}{|A|} .
\end{aligned}
$$

THEOREM 1. A semigroup $S$ satisfies (SFC) if and only if there is a net of arithmetic means on $S$ which converges in norm to left invariance.

Proof. In view of Lemma 1 , necessity is obvious. Suppose, on the other hand, that $S$ satisfies (SFC). Let $\mathcal{F}$ be the family of all finite subsets of $S$ directed upward by inclusion and let $E=(0,1)$ directed (towards 0 ) by its usual ordering. Let $D$ be the product directed set $\mathcal{F} \times E$. If $d=(F, \alpha) \in D$, then there exists a finite set $A_{d} \subset S$ such that

$$
\left|A_{d} \sim s A_{d}\right| /\left|A_{d}\right|<\alpha
$$

for all $s \in F$. It is clear from Lemma 1 that $\left\{\mu_{\boldsymbol{A}_{d}}\right\}$ converges in norm to left invariance.

3. Semigroups satisfying (SFC). In view of the discussion in $\$ 2$ it is natural to ask: Does every left amenable semigroup satisfy (SFC)? An affirmative answer would be very pleasant indeed, and we prove some results which point in this direction.

THEOREM 2. Every left cancellative left amenable semigroup satisfies (SFC).

Proof. This is evident from the discussion in $\$ 2$.

THEOREM 3. Every finite left amenable semigroup satisfies (SFC).

Proof. A finite semigroup $S$ is left amenable if and only if it contains a unique minimal right ideal $R$ (see [1] or [8]). In this situation we must have $s R=R$ for all $s \in S$ (see [2]) and thus (SFC) holds (take $A=R$ ).

The next result is less trivial. Keep in mind that every commutative semigroup is amenable. 


\section{THEOREM 4. Every commutative semigroup satisfies (SFC).}

Proof. Let $S$ be a commutative semigroup. We define a relation $R$ on $S$ by: $s R t$ if and only if there exists $u \in S$ such that $s u=t u$. It is easy to see that $R$ is a congruence ${ }^{3}$ on $S$ (see [5] for details) and the quotient semigroup $S^{\prime}=S / R$ is commutative and cancellative. Theorem 2 implies that $S^{\prime}$ satisfies (SFC). The remainder of the proof is a construction showing that (SFC) can be "lifted" from $S^{\prime}$ to $S$.

If $x \in S$ we let $x^{\prime}$ denote the image of $x$ under the natural mapping of $S$ on to $S^{\prime}$. Given a finite set $F \subset S$ and $\epsilon>0$, there exists a finite set $A^{\prime} \subset S^{\prime}$ such that

(1) $\left|A^{\prime} \sim x^{\prime} A^{\prime}\right|<\epsilon\left|A^{\prime}\right|$ for all $x \in F$.

Let $A$ be a set of representatives for the elements of $A^{\prime}$. It could conceivably happen that

(2) whenever $x \in F, a, b \in A$, and $x^{\prime} a^{\prime}=b^{\prime}$ then $x a=b$ also. If this is the case, then $|A \sim x A|=\left|A^{\prime} \sim x^{\prime} A^{\prime}\right|$ for all $x \in F$ and so

$$
|A \sim x A|<\epsilon|A| \quad \text { for all } x \in F .
$$

We cannot, of course, expect to be so fortunate. However, it is possible to obtain this situation by modifying $A^{\prime}$ and $A$ as follows.

Let $A^{\prime}$ and $A$ be as above, and let $x_{i}^{\prime} a_{i}^{\prime}=b_{i}^{\prime}(i=1,2, \cdots, m)$ be an enumeration of the relations of the form $x^{\prime} a^{\prime}=b^{\prime}$ which hold with $x \in F, a, b \in A$. Since $x_{1}^{\prime} a_{1}^{\prime}=b_{1}^{\prime}$, there is an element $u_{1} \in S$ such that $x_{1} a_{1} u_{1}=b_{1} u_{1}$. Taking $A_{1}^{\prime}=\left\{a^{\prime} u_{1}^{\prime}: a \in A\right\}$ and $A^{\prime}=\left\{a u_{1}: a \in A\right\}$ we have $\left|A_{1}^{\prime}\right|=\left|A^{\prime}\right|$ and $\left|A_{1}^{\prime} \sim x^{\prime} A_{1}^{\prime}\right|=\left|A^{\prime} \sim x^{\prime} A^{\prime}\right|$ for all $x \in F$ because $S^{\prime}$ is (right) cancellative. Thus $A_{1}^{\prime}$ satisfies (1) and, in addition, we have $x_{1}\left(a_{1} u_{1}\right)=b_{1} u_{1}$. Now $x_{2}^{\prime} a_{2}^{\prime} u_{1}^{\prime}=b_{2}^{\prime} u_{1}^{\prime}$ and so there is an element $u_{2} \in S$ such that $x_{2} a_{2} u_{1} u_{2}=b_{2} u_{1} u_{2}$. Taking $A_{2}^{\prime}=\left\{a^{\prime} u_{1}^{\prime} u_{2}^{\prime}: a \in A\right\}$ and $A_{2}$ $=\left\{a u_{1} u_{2}: a \in A\right\}$ we have $\left|A_{2}^{\prime}\right|=\left|A^{\prime}\right|$ and $\left|A_{2}^{\prime} \sim x^{\prime} A_{2}^{\prime}\right|=\left|A^{\prime} \sim x^{\prime} A^{\prime}\right|$ for all $x \in F$. Thus $A_{2}^{\prime}$ satisfies (1) and, in addition, we have $x_{i}\left(a_{i} u_{1} u_{2}\right)$ $=b_{i} u_{1} u_{2}$ for $i=1,2$. The induction is now clear. By repeating this process $m$ times we obtain a set $A_{m}^{\prime} \subset S^{\prime}$ satisfying (1) and a set $A_{m}$ of representatives satisfying (2). This is the desired situation.

The above proof could be simplified slightly, but we have arranged the presentation in order to make it clear to what extent the constructions are valid in the noncommutative case. First of all, we should mention that the essential fact needed to show that $R$ is a congruence is that the family of (right) ideals in $S$ has the finite intersection property. Hence the construction of $S^{\prime}$ makes sense for (noncommutative) semigroups having the finite intersection property

${ }^{3}$ We follow the terminology of [3]. A congruence is an equivalence relation $R$ with the property that $x R y$ implies $a x R a y$ and $x a R y a$ for all $a \in S$. 
on right ideals (every left amenable semigroup has this property), and in this case $S^{\prime}$ is right cancellative. The next essential thing is that $S^{\prime}$ should satisfy (SFC). This implies, of course, that $S^{\prime}$ is left amenable and hence $S$ is left amenable also (see [9]). The remainder of the proof goes through without change. Thus we have

THEOREM 5. If $S$ is left amenable and if $S^{\prime}$ satisfies (SFC) then $S$ satisfies (SFC).

The above theorem is interesting in light of the following conjecture of Sorenson: Any right cancellative left amenable semigroup must be left cancellative also (see [6] for a discussion of Sorenson's conjecture).

THEOREM 6. If Sorenson's conjecture holds, then every left amenable semigroup satisfies (SFC) and hence admits a net of arithmetic means converging in norm to left invariance.

Proof. If $S$ is left amenable then, as remarked above, the construction of $S^{\prime}$ is valid and $S^{\prime}$ is right cancellative and left amenable. If Sorenson's conjecture is valid then we can conclude that $S^{\prime}$ is also left cancellative and hence satisfies (SFC).

\section{REFERENCES}

1. L. N. Argabright, A note on invariant integrals on locally compact semigroups, Proc. Amer. Math. Soc. 17 (1966), 377-382.

2. A. H. Clifford, Semigroups containing minimal ideals, Amer. J. Math. 70 (1948), 521-526.

3. A. H. Clifford and G. B. Preston, The algebraic theory of semigroups, Math. Surveys No. 7, Amer. Math. Soc., Providence, R. I., 1961.

4. M. M. Day, Amenable semigroups, Illinois J. Math. 1 (1957), 509-544.

5. E. Granirer, $A$ theorem on amenable semigroups, Trans. Amer. Math. Soc. 111 (1964), 367-379.

6. - Extremely amenable semigroups. II (to appear).

8. W. G. Rosen, On invariant means over compact semigroups, Proc. Amer. Math.

7. I. Namioka, Følners condition for amenable semigroups, Math. Scand. 15 (1964), Soc. 7 (1956), 1076-1082.

9. C. O. Wilde and L. N. Argabright, Invariant means and factor-semigroups, Proc. Amer. Math. Soc. 18 (1967), 226-228.

UNIVERSITY OF MINNESOTA 